\title{
Die Wahrnehmung der Pädagogik Johann Friedrich Herbart in Polen
}

\section{Perceiving the pedagogy of Johann Friedrich Herbart in Poland}

\begin{abstract}
The article shows the development of Polish interests in the pedagogical mind of J. F. Herbart. In this work we chronologically presented the most important Polish publications on Herbart we drew attention to the change of its perception in different historical periods. There is no doubt that a large part of Polish authors esteem the educational Herbart and tried to popularize his ideas among Polish teachers. It is understood that the greatest interest in 'herbartyzmem' was in the nineteenth century but it declined sharply in the next century. This was associated with the development of new educational systems. The article is an important contribution to a better understanding of the reception of the German educational thought in other countries.
\end{abstract}

\section{Keywords}

Herbartyzm, pedagogical thought, history of education.

In der Weltgeschichte mangelte es nicht an außerordentlichen Denkern, die eine große Rolle in der Entwicklung der Theorie und Praxis von Erziehung und Lehre spielten. Zu ihnen zählte ohne Zweifel Johann Friedrich Herbart (1776-1841) - ein hervorragender deutscher Philosoph, Psychologe und Pionier der Wissenschaftspädagogik. Erziehungshistoriker zählen ihn zu den hervorragendsten Klassikern des neueren Pädagogikgedankens neben deren 
Gründern, wie zum Beispiel John Locke, Johann Amos Comenius, Jean-Jacques Rousseau, Johann Heinrich Pestalozzi, Herbert Spencer oder John Dewey. Es ist unbestritten, dass die von Herbart popularisierten Bildungsideen Erkenntnisse waren, die von Pädagogen aus anderen Ländern aufgegriffen wurden und auf die Gestaltung der heimatlichen Erziehungspraktiken Einfluss nahmen. Für die herbartische Pädagogiktheorie interessieren sich auch die Polen. Ein Beweis hierfür ist der Reichtum an fachspezifischer Literatur, welche sich auf das Werk des deutschen Pädagogen bezieht. Er bestätigt, dass das Interesse sich nicht nur auf die herbartistisch Reflexion begrenzt, sondern dass auch die Übernahme einiger seiner Erkenntnisse stattfand und man versuchte, die didaktischen Postulate und herbartische Erziehung an polnischen Schulen einzuführen.

Herbart wurde nicht nur als Pädagoge, sondern auch als Philosoph berühmt. In seinen pädagogischen Überlegungen basierte er auf der Ethik und der Psychologie. Er war der Meinung, dass Ethik (die Lehre von der moralischen Schönheit) das Erziehungsziel bestimmt. Herbart ist Schöpfer der Theorie des erziehenden Unterrichts, also eines Unterrichts, der nicht nur den Schüler um Wissen bereichert, sondern auch seinen Charakter weiterbildet. Den Verdienst Herbarts und seiner Schüler stellt die Weiterentwicklung der Ideen in Bezug auf wirksame Unterrichtsmethoden dar. Sie führten in die Pädagogik die so genannte Theorie der formalen Stufen ein, die - wie sie behaupteten - eine wirksame Grundlage des Unterrichts bilden. Herbart hat die Unterrichts- und Erziehungstheorie „verwissenschaftlicht“, und mit seiner pädagogischen Tätigkeit gab er einen Impuls zu nicht endenden Diskussionen über den Unterrichtsund Erziehungsprozess. Diese Diskussionen wurden in verschiedenen Ländern geführt. Sowohl Theoretiker, als auch Praktiker der Erziehung und des Unterrichts nahmen an ihnen teil. Ihr Ergebnis waren unter anderen Artikel und Bücher, die zweifelsfrei die pädagogische Literatur bereicherten.

Verfolgen wir deshalb den Inhalt der wichtigsten, auf Lösungen für Herbarts Bildungssystem bezogenen polnischen bildungshistorischen Publikationen. Dabei soll es hier nicht nur um wissenschaftliche Bücher gehen, sondern auch um allgemeinbildende Zeitschriften, Enzyklopädien und erziehungshistorische Lehrbücher, die studierende Jugendliche verwenden. Die polnische Literatur, die den Bildungsgedanken von Herbart behandelt, ist sowohl in Bezug auf die Anzahl, als auch auf die Qualität reichhaltig. Ab der zweiten Hälfte des neunzehnten Jahrhunderts bis hin zum jetzigen Zeitpunkt analysierten Bildungshistoriker und Pädagogen vielseitig die Rätsel, die mit der herbartischen Pädagogik verbunden sind. Unter den Autoren waren erwiesene Kenner der 
Bildungsgeschichte, aber auch Forscher, die sich mit den pädagogischen Gedanken des neunzehnten Jahrhunderts in kleinen Schritten beschäftigten. Ihre Publikationen sind interessante Wissensquellen über Herbart, die zeigen, wie die Polen in bestimmten historischen Perioden seine pädagogischen Idee verstanden, wie sie nach den Empfehlungen des deutschen Pädagogen, das vier Etappen umfassende Lernen und Signalisieren von Klarheit mit etwas in Verbindung bringen, nach System und Methode, agierten.

Inmitten der betreffenden Publikationen über Herbart mangelt es an der Behandlung der vorgestellten Entwicklung des Interesses am Herbartizismus in Polen. Für ein besseres Verständnis der Spezifika, dieses Interesses und der Vorgänge der Übernahme der pädagogischen Ideale ist diese auf reichhaltigen historischen Quellen beruhende Zusammenfassung unabdingbar. Wichtig ist außerdem die Darstellung der Ergebnisse früherer Forschungen am Herbartizismus. Ziel dieses Artikels ist die allgemeine Charakterisierung polnischer, die bildungserzieherische Konzeption Herbarts betreffenden, historisch-pädagogischer Schriftstücke. Ziel des Artikels ist es nicht, Publikationen zu diesem Thema einfach aufzuzählen, sondern auf die Wahrnehmung des herbartischen Bildungsgedanken durch polnische Autoren und die Erscheinung, welche Elemente dieses Gedanken besonders geschätzt wurden und warum, aufmerksam zu machen. Die Entwicklung pädagogischer Schriften und die Übernahme fremder Bildungsideale waren von unterschiedlichen Faktoren und besonders von der politischen Situation in Polen abhängig.

\section{Herbart in der polnischen Pädagogik in der Periode der nationalen Unfreiheit}

In der Geschichte der Erkundung des pädagogischen Schaffens von Herbart durch die Polen können wir einige Perioden unterscheiden. ${ }^{1}$ Die erste von ihnen fiel in eine schwierige Etappe des Lebens der polnischen Nation. Am Ende des achtzehnten Jahrhunderts vollzogen Russland, Preußen und Österreich eine Teilung Polens, und der Staat hörte für über 120 Jahre auf zu existieren. In der Phase der polnischen Unfreiheit nahm die Bildung eine beschämende Entwicklung, das Schulsystem war russifiziert und germanisiert. Trotz der

\footnotetext{
${ }^{1}$ J. Dybiec, Recepcja idei pedagogicznych $w$ Polsce, in: Recepcja w Polsce nowych kierunków i teorii naukowych, ed. A. Strzałkowski, Kraków 2001, p. 195 i n.
} 
schwierigen Bedingungen für Lehre und Bildung wurde strebsame Arbeit verrichtet, die sich auf die Verbesserung des Ausbildungsprozesses und der Erziehung sowie auf den Kampf um die Erhaltung der nationalen Identität konzentrierte. In diesen nicht einfachen Zeiten begrenzten sich die Polen nicht nur auf die eigene Schreibtätigkeit, sondern sie interessierten sich auch lebhaft für die pädagogischen Ideale ausländischer Wissenschaftler. Ihre Werke wurden im Original gelesen, ins Polnische übersetzt und man knüpfte an deren Inhalte in Publikationen an.

In der Zeit, in der Herbart lebte und wirkte, gewann sein Pädagogikgedanke nur wenige Befürworter im eigenen Land und in den Nachbarländern. ${ }^{2}$ Seine Vorlesungen in Königsberg hörte ein polnischer Emigrant - Bronisław Ferdynand Trentowski, der spätere Autor des detaillierten pädagogischen Werkes unter dem Titel Chowanna. ${ }^{3}$ Die von Herbart veröffentlichen Thesen waren einige von vielen, für die sich Trentowski, sein philosophisch-pädagogisches System aufbauend, interessierte. Dennoch stand nicht die Wissenschaftspädagogik von Herbart, sondern die idealistische deutsche Philosophie im Zentrum seines Interesses. In der Epoche der Spätromantik begann ein anderer deutscher Pädagoge - Friedrich Adolf Diesterweg - bedeutenden Einfluss auf die polnische Pädagogik auszuüben. ${ }^{4}$ In einer Phase des Positivismus unter den polnischen Pädagogen und besonders unter den im russischbesetzten Gebiet wirkenden, interessierte sie ein englischer Pädagogikgedanke, dessen damals hervorragendster Vertreter Herbert Spencer war. ${ }^{5}$ Veröffentlicht wurden damals zahlreiche Übersetzungen fremdsprachiger pädagogisch-psychologischer Schriften, ${ }^{6}$ die im Gegenzug bedeutend für die polnische Pädagogikliteratur waren.

${ }^{2}$ F. Majchrowicz, Historia pedagogii dla użytku seminariów nauczycielskich $i$ nauki prywatnej, t. 2, Lwów 1920, p. 74.

${ }^{3}$ B. F. Trentowski, Chowanna, czyli system pedagogiki narodowej jako umiejętności wychowania, nauki i oświaty, słowem wykształcenia naszej młodzieży, t. 1-2, ed. A. Walicki, Wrocław-Warszawa-Kraków 1970.

${ }^{4}$ M. Krupa, Fryderyk Adolf Diesterweg i jego wpływ na polską pedagogike w XIX wieku, Wrocław 1976.

${ }^{5}$ B. Nawroczyński, Polska myśl pedagogiczna, Lwów -Warszawa 1938; M. Sękowski, Spencer i pozytywizm warszawski, Lublin 1986.

${ }^{6}$ J. Dybiec, Polska w orbicie wielkich idei: polskie przekłady obcojęzycznego piśmiennictwa 1795-1918, t. 1, Warszawa 2011. 
Intensivere Interessen für den Herbartizismus kamen inmitten der polnischen Pädagogen am Ende des neunzehnten Jahrhunderts zum Vorschein.? Die Pädagogik von Herbart nahm sich Henryk Wernic - Autor bedeutender Arbeiten, darunter der Gedanken über Erziehung und Unterricht ${ }^{8}$ - zum Vorbild. Das Wissen über neue Trends in der Pädagogik erwarb er während eines einige Jahre dauernden Aufenthalts in Dresden, sowie in Paris und London. Wernic wollte die Pädagogik - genau wie Herbart - auf Ethik und Psychologie stützen, er empfahl die Praktizierung von Lernregeln für den Erzieher. ${ }^{9}$ Man ist bemüht, Herbarts pädagogische Postulate für die Ausbildung der polnischen Lehrer zu nutzen. Publikationen über didaktische Inhalte und die erzieherische Vorbereitung für Lehrer enthalten Elemente des Pädagogikgedankens des deutschen Wissenschaftlers. ${ }^{10}$ Ein Beispiel dessen kann unter anderem die Didaktik von Mieczysław Baranowski ${ }^{11}$ sein, oder der Text zur Pädagogikvorlesung von Władysław Seredyński. ${ }^{12}$ Wie Baranowski behauptet, äußert sich der Herbartizismus im „absoluten Anwenden von Regeln zum Unterrichten des Erziehers. Darüber hinaus übernimmt er Verantwortung für den Verlauf der Unterrichtsstunde im Rahmen von Herbarts formalen Schritten"13. Die Pädagogik nach Herbart fand in der Bildung in polnischen Mittelschulen in Galizien Anwendung, das ein Teil der österreichischen Monarchie war. Österreichische Schulen sind für die intensive Verwendung des Herbartizismus berühmt. ${ }^{14}$

7 D. Stępkowski, Herbart i jego myśl w Polsce. Dzieje recepcji i oddziaływań, „Artes liberales. Zeszyty Naukowe Akademii Humanistycznej im. A. Gieysztora" (2007), no 1, pp. 1-33; J. Gara, Specyfika polskiej recepcji twórczości Johanna Friedricha Herbarta, „Przegląd Pedagogiczny” (2012), no 2, pp. 22-32.

${ }^{8} \mathrm{H}$. Wernic, Myśli o wychowaniu i nauczaniu. Z autobiografia i portretem autora, Warszawa 1895.

9 S. Wołoszyn, Nauki o wychowaniu w Polsce w XX wieku. Próba syntetycznego zarysu na tle powszechnym, Kielce 1998, p. 17.

${ }^{10}$ C. Majorek, System kształcenia nauczycieli szkół ludowych w Galicji doby autonomicznej 1871-1914, Wrocław 1971.

${ }_{11}$ M. Baranowski, Dydaktyka do użytku seminariów nauczycielskich i nauczycieli szkót wyższych, Lwów 1895.

12 W. Seredyński, Tekst do wykładu pedagogiki. Wykład metody nauk realnych $w$ szkole ludowej dla użytku seminariów nauczycielskich, Tarnów 1888.

13 S. I. Możdżeń, Historia wychowania 1795-1918, Sandomierz 2006, s. 333.

14 S. Kot, Historia wychowania, t. 2, Lwów 1934, s. 160. 
Im Jahre 1899 veröffentlichte Tadeusz Troskolański - einer der in der Mittelschule arbeitenden Lehrer - ein populärwissenschaftliches Buch unter dem Titel Herbarts Verdienste rund um die Erziehung. Das Werk glorifiziert beinahe kritiklos die Grundsätze der Herbartischen Pädagogik. ${ }^{15}$ In dieser Zeit verbreitete Leon Kulczyński Wissen über Herbart durch die Erziehungsenzyklopädie, welche reichhaltige Informationen über das Leben des deutschen Pädagogen und seine Pädagogiktheorie enthaltende, detaillierte biografische Losungen herausgab. Seine Betrachtungen beendend, schrieb er: „Die Herbartpädagogik findet immer breitere Anerkennung [...] der Theoretiker oder direkt von denen sich zu seinen Anhängern zählenden, oder derer, die trotz des Widerstandes gegen ihn nicht zögern, die Tragweite seiner Verdienste zu erkennen und seinen Gedanken ordnungsgemäß zu übernehmen." ${ }^{\prime 16}$ Im angesprochenen Zeitraum war der größte Liebhaber des Pädagogikgedankens von Herbart Antoni Danysz ein Erziehungstheoretiker, klassischer Philologe und Professor der Lemberger Universität, Autor origineller Publikationen aus dem Bereich der Erziehung ${ }^{17}$ und Didaktik. Den Leitgedanken von Herbarts Pädagogiktheorie charakterisierend zeigte er zu naiv, dass sein Einfluss „so stark [ist], dass er auf die öffentliche Erziehung eines großen Teils der europäischen Nationen einwirkt". ${ }^{18}$ Anhänger der Theorie von Herbart waren in dieser Epoche auch solche Autoren, wie der Professor der Lemberger Universität Bolesław Mańkowski oder der Direktor von Mittelschulen in Galicien, Franciszek Majchrowicz.

Die erste Ausgabe des Werks von Herbart unter dem Titel Allgemeine Pädagogik abgeleitet aus dem Zweck der Bildung erschien in polnischer Übersetzung erst im Jahre 1912. Die Übersetzung fertigte Teodor Stera an und begann das Werk mit einer kurzen Einleitung. Er stellte den deutschen Autor als „Philosoph unter den Pädagogen und Pädagoge unter den Philosophen“ vor und äußerte die Ansicht, dass er bisher unter den polnischen Lesern wenig bekannt sei. Der Übersetzer begründete die Notwendigkeit der Ausgabe des Buches von Herbart mit den Worten: „Herbarts Philosophie hat schon ihres getan; heute ist sie, einen kleinen Kreis von Anhängern und Weiterführern ausgenommen, eher wenig bekannt und anerkannt, doch in Sachen Erziehung

15 T. Troskolański, Zasługi Herbarta około wychowania, Lwów 1899.

${ }^{16}$ L. Kulczyński, Herbart Jan Fryderyk, in: Encyklopedia wychowawcza, t. 5, Warszawa 1901, p. 275.

17 A. Danysz, O wychowaniu, Lwów 1903.

18 A. Danysz, O kształceniu, Lwów 1918, p. 10. 
ist der Gedanke ebenso bedeutsam wie fruchtbar. Dies sagte bisher niemand". ${ }^{19}$ Für den Übersetzer war es selbstverständlich, dass eine Notwendigkeit der Übersetzung von Herbarts Arbeiten ins Polnische und des Kommentierens und der Analyse ihrer Inhalte besteht. In der Zeitschrift „Erziehung zu Hause und in der Schule" wurde mit Hochachtung über den Inhalt der Publikation der Werke Herbarts in polnischer Sprache geschrieben: „<<Der größte Pädagoge unter den Philosophen und der größte Philosoph unter den Pädagogen $>>$ wie sie ihn in Deutschland nennen, bildet eine Epoche im Wirken der Erziehungstheorie. In seinen Werken findet der Leser nicht nur Ideen und Ansichten - wie bei früheren Pädagogen - aber auch ihre Unterstützung für bestimmte beschriebene, vollkommen psychologische Grundlagen, er findet in ihnen zahlreiche frische Gedanken, mit deren Weiterentwicklung sich die gegenwärtige Pädagogik befasst" ${ }^{20}$ Die polnische Ausgabe der allgemeinen Pädagogik Herbarts rief eher kein großes Interesse bei den Kritikern der neuen Ausgaben hervor. Davon zeugt die verschwindend geringe Zahl inhaltlicher Rezensionen dieses Werkes, die auf den Seiten damaliger Zeitschriften veröffentlicht wurden.

Die zweite Hälfte des neunzehnten Jahrhunderts und einige Jahre vor dem Ersten Weltkrieg waren eine Periode, in der der Herbatizismus bei polnischen Pädagogen die größte Anerkennung weckte. Es gab damals einen bedeutenden Bedarf an neuen Inhalten, die es ermöglichten, im Prozess der Bildung und Erziehung genutzt zu werden. Die übertriebene Faszination vom Herbartizismus erlaubte einigen Autoren nicht immer eine objektive Bewertung seines Wertes in der Bildung und Erziehung. Unter den polnischen Arbeiten über Herbart aus dieser Epoche fehlte es nicht an Urteilen, auf welchen ein Brandmal der Hagiografie lastet. Die Vertreter der neuen Pädagogikrichtungen kritisierten „die Einseitigkeit der traditionellen Pädagogik”. ${ }^{21}$ Für die modernen Strömungen, darunter u.a. die individualistische Pädagogik, den pädagogischen Soziologismus, pädagogischen Personalismus oder die Kulturpädagogik interessierten sich die polnischen Pädagogen immer lebhafter. Es verringerte sich unter ihnen dagegen das Interesse an damaligen Bildungs- und Erziehungskonzepten.

19 J. F. Herbart, Pedagogika ogólna wywiedziona z celu wychowania, tł. T. Stera, Warszawa 1912, p. VI.

20 Z., recenzja, „Wychowanie w Domu i Szkole” (1912), t. 2, pp. 154-155.

${ }^{21}$ K. Sośnicki, Rozwój pedagogiki zachodniej na przełomie XIX i XX wieku, Warszawa 1967, pp. 46-47. 


\section{Zwischenkriegszeit}

Im Jahre 1918 erlangte Polen seine Unabhängigkeit nach vielen Jahren der Unfreiheit und begann, die Fundamente eines neuen Staates aufzubauen, in welchem Erziehung und Bildung eine große Rolle spielen sollten. Die Unabhängigkeit von den Besatzungsmächten schaffte bessere Bedingungen für die Entwicklung der Bildung und die Äußerung eigener Gedanken und Überzeugungen. ${ }^{22}$ Das bedeutet jedoch nicht, dass polnische Pädagogen das Wirken ausländischer Pädagogikklassiker ignorierten und sich nicht für die neuesten Bildungserrungenschaften in anderen Ländern interessierten. In den 20er und 30er Jahren des vergangenen Jahrhunderts wurden ausländische Pädagogikliteratur und originale Protokollschriften, welche dem Leser das Wesen der Ideale und Ziele der Erziehung und Lehre, die in der Vergangenheit von Herbart und seinen Schülern propagierten, näher brachten, ins Polnische übersetzt. Die Ideale waren dennoch damals schon nicht mehr von so großer Priorität, wie für die polnischen Pädagogen im neunzehnten Jahrhundert. Die Doktrin der Herbartisten wurde den Lehrern nicht mehr durch die Schulbehörde aufgedrängt. ${ }^{23}$ Immer weiter interessierten sie sich für Thesen, die durch die Gründer der sogenannten neuen Erziehungswissenschaftsbewegung ausgegeben wurden. ${ }^{24}$

Die Schöpfer der polnischen bildungshistorischen Literatur in der Zwischenkriegszeit zeigten, dass Herbart großen Einfluss auf die theoretische Entwicklung des Pädagogikgedankens hatte. Geringer war dagegen sein Einfluss auf die damalige Schulpraxis. ${ }^{25}$ In der Pädagogikliteratur wurde bemerkt, dass die Herbartisten eine zu große Aufmerksamkeit auf die durch sie erarbeiteten Theorien des Verlaufes der Lehre und der formalen Schritte wenden. ${ }^{26}$ In der Zwischenkriegszeit wurde Herbart durch die polnischen Popularisatoren des pädagogischen Wissens als pädagogischer Klassiker behandelt und auf seine Theorie beriefen sich Vertreter unterschiedlicher Pädagogikrichtungen. Auf die

${ }^{22}$ W. Szulakiewicz, Historia oświaty i wychowania w Polsce 1918-1939. Studium historiograficzne, Toruń 2000.

23 S. I. Możdżeń, Historia wychowania 1795-1918, s. 62.

${ }^{24}$ J. Sobczak, Recepcja idei nowego wychowania w polskiej pedagogice okresu między wojnami, Bydgoszcz 1979.

25 F. Kierski, Podręczna encyklopedia pedagogiczna, t. 1, Lwów-Warszawa 1923, pp. 145-147.

${ }^{26}$ B. Nawroczyński, Zasady nauczania, Lwów-Warszawa 1930, p. 313. 
Erziehungsidee von Herbart bezog sich der Dominikaner Jacek Woroniecki, ${ }^{27}$ Autor des Werkes mit dem Titel Katholische Erziehungsethik, ${ }^{28}$ in welchem er begründet, dass die Pädagogik ein Teil der Ethik ist und keine eigenständige Wissenschaft. Die Schwäche der Erziehungstheorie und Erziehungspraxis sah er in der von ihm bevorzugten Dominanz des moralischen Intellektualismus ${ }^{29}$. Herbarts Pädagogikgedanke weckte ebenso die Neugierde bei Lucjan Zarzecki einem polnischen Theoretiker der nationalen Erziehung. ${ }^{30}$ Er konzentrierte sein Interesse auf Herbarts Betrachtungen der Formung starker Charaktere bei Jugendlichen.

In den dreißiger Jahren des vergangenen Jahrhunderts war seine Publikation Umriss pädagogischer Vorlesungen ein wichtiges Ereignis, das Einfluss auf die Erkundung der pädagogischen Konzepte von Herbart hatte. Das Werk wurde nur als Auflage der wissenschaftlichen Pädagogikgesellschaft veröffentlicht. Die polnische Version wurde auf Grundlage der zweiten deutschen Ausgabe, die von Otto Willmann und Theodor Fritzsch ${ }^{31}$ neuaufgelegt wurde, erstellt. Die polnische Diese wurde nicht mit einer wissenschaftlichen Einleitung versehen, welche ein Bild der Leistungen Herbarts geben würde. Teilweise erwiesen sich ihre Rezensionen, die auf den Seiten der Zeitschriften besprochen wurden, als Inhalt der neuen Ausgabe. Mirosław Sekreta bemerkte in „Chowannie”, dass der gegenwärtige Pädagogikgedanke gegenüber der Pädagogik Herbarts , am klarsten und eindeutigerer im Widerstand" [ist]. Es stören ihn der individualistische Charakter im Erziehungsziel der herbartischen Pädagogik, er verurteilt die Begrenzung in der Erziehung auf die Entwicklung des Intellektes, aus der Störung von Befinden und Willen, entwirft er das Dogma der formalen Stufen". ${ }^{32}$ Für Sekreta war es natürlich, dass Herbart, trotz der Unvollkommenheit einiger seiner Annahmen über Bildung und Erziehung, zur Entwicklung der Pädagogiktheorie beitrug. Wie in der Zeitschrift „Freund der Schule” bewertet, zeigt sich, dass sich aktuell einige pädagogische Grundsätze Herbarts desaktualisiert haben. Es

${ }^{27}$ J. Kostkiewicz, Kierunki i koncepcje pedagogiki katolickiej w Polsce 1918-1939, Kraków 2013, p. 100.

${ }^{28}$ J. Woroniecki, Katolicka etyka wychowawcza, Poznań-Warszawa-Wilno-Lublin 1925.

29 S. I. Możdżeń, Historia wychowania 1918-1945, Sandomierz 2006, p. 36.

${ }^{30}$ L. Zarzecki, Charakter jako cel wychowania, Warszawa 1918, pp. 62-63.

${ }^{31}$ J. F. Herbart, Wykłady pedagogiczne w zarysie, tł. J. Jakóbiec, przejrzał F. Bielak, Warszawa 1937, p. 3.

32 M. Sekreta, recenzja, „Chowanna” (1937), t. 7, pp. 376-380. 
ist nötig, sie dennoch anzuerkennen, da sie eine große Rolle in der Geschichte der Erziehungswissenschaft spielten. ${ }^{33}$

In der polnischen pädagogisch-historischen Literatur der Zwischenkriegszeit überwiegt die Meinung, dass die ethischen Ansätze und die psychologische Pädagogik Herbarts „,sich als unbegründet erwiesen”. ${ }^{34}$ Am Ende der Zwischenkriegszeit bewerte Ludwik Chmaj den Herbatizismus in seinem Buch Richtungen und Strömungen der gegenwärtigen Pädagogik streng, als „alte Schule des neunzehnten Jahrhunderts“, die „sich als universeller Intellektualismus, sowie feindlich in Bezug auf die Individualität des Kindes kennzeichnet“. Chmaj behauptete, dass der Erziehungsprozess in der Herbartischen Schule mechanisch, übertriebene Aufmerksamkeit, nicht auf das Lernen oder auf den Lehrer, sondern nur auf das zu lernende Material und die Methode ausrichtend, ausübte. Der Autor erinnerte, dass in Deutschland vor Ende des neunzehnten Jahrhunderts starke Oppositionen gegen den Formalismus der Herbartischen Schule erschienen waren, was im angesprochenen Beispiel die Ausgabe von Lindis Buch Persönlichkeitspädagogik. Warnung vor dem zeitgenössischen Vertrauen in die Methoden ${ }^{35}$ aus dem Jahre 1896 war.

Polnische Pädagogen der Zwischenkriegszeit äußerten sich kritisch zu einigen Postulaten der Herbartischen Didaktik, zu einer zu großen Aufmerksamkeit, die auf humanistische Fächer gelegt wurde, die eine führende Rolle in der Lehre des Erziehenden erfüllen sollten. ${ }^{36}$ Wie bereits erwähnt wurde, verlor im Verlauf der Jahre der Pädagogikgedanke von Herbart an Attraktivität. Bis zum Jahre 1939 war der am meisten übersetzte deutsche Pädagoge Friedrich Wilhelm Foerster ${ }^{37}$. In der Zwischenkriegszeit wurden ebenso die Bücher von Georg Kerschensteiner ins Polnische übersetzt. Seine Konzeption der Arbeitsschulen interessierte Pädagogen, die die pädagogischen Richtungen analysierend die Idee der sogenannten neuen Bewegung der Erziehungswissenschaft propagierten. ${ }^{38}$

${ }^{33}$ W. Osuch, recenzja, „Przyjaciel Szkoły” (1937), no 8, pp. 306-307.

${ }^{34}$ S. Kot, Historia wychowania, t. 2, Lwów 1934, p. 161.

${ }^{35}$ L. Chmaj, Kierunki i prądy pedagogiki wspótczesnej, Warszawa-Wilno 1938, pp. 135-137.

${ }^{36}$ K. Sośnicki, Dydaktyka ogólna, in: Encyklopedia wychowania, t. 2, Warszawa 1937, p. 76.

${ }^{37}$ D. Koźmian, Chrześcijańska myśl wychowawcza Fryderyka Wilhelma Foerstera i jej recepcja $w$ Polsce, Szczecin 1996.

38 J. Wnęk, Polskie przekłady literatury zagranicznej 1918-1939, Kraków 2006. 


\section{In der Volksrepublik Polen 1945-1989}

Nach dem Zweiten Weltkrieg befand sich Polen in der sowjetisch beeinflussten Zone und musste die Lehre und Bildung betreffenden Richtlinien Moskaus umsetzen. Besonders schwierig für das Schulwesen und die wissenschaftlichen Forschungen waren die Jahre 1949-1956, die Phase des sogenannten Stalinismus, als in Polen mit reger Intensivität das Grundgerüst des Sozialismus aufgebaut wurde. ${ }^{39}$ Die westeuropäische und amerikanische Pädagogik wurde damals mit dem Attribut bürgerlich beschrieben. Dem gegenüber stand das die Ideale der kommunistischen Erziehung propagierende Modell der sowjetischen Pädagogen. Die komplizierte Situation hielt die Historiker und Pädagogen nicht vom Studium der richtigen Pädagogikgedanken, darunter dem deutschen, ab. In der Verbreitung des pädagogischen Wissens spielte die Bibliothek der Pädagogikklassiker - ausgegeben durch das Komitee für Pädagogikwissenschaften der polnischen Wissenschaftsakademie - eine führende Rolle. Die Ausgabe der Serie stellte das Leben und Schaffen eines Dutzends der größten Schöpfer der Pädagogiktheorie dar. Ihr Vorteil war es, dass die Vorwörter und Kommentare zu den veröffentlichten Büchern die damals kompetentesten Autoren schrieben.

In der Epoche der Volksrepublik Polen waren die Wissenschaftler, die die Pädagogikgedanken untersuchten, einem großen ideologischen Druck ausgesetzt. Es existierte die Mode, die literarischen Annahmen der Autoren zu untersuchen, die ihr Erziehungsprogramm mit Bestrebungen zum gesellschaftlichen Umbau verbanden. Für solche Autoren wurden unter anderem Comenius, Rousseau oder Karl Marx gehalten. ${ }^{40}$ Einige polnische Erziehungstheoretiker übernahmen kritiklos den sowjetischen Pädagogikgedanken, bewerteten ihn, und besonders die Kollektiverziehung von Anton Makarenko, hoch. ${ }^{41}$ In der polnischen bildungshistorischen Literatur dieser Zeit wurde die Neuartigkeit der Bildungsgrundsätze Georg Kerschensteiners ${ }^{42}$ unterstrichen.

39 W. Szulakiewicz, Historia oświaty i wychowania w Polsce 1944-1956, Kraków 2006.

${ }^{40}$ Z dziejów myśli pedagogicznej. Materiały do studiów, eds. B. Suchodolski, W. Okoń, Warszawa 1958, p. 8.

${ }^{41}$ M. Łobocki, Teoria wychowania w zarysie, Kraków 2010, p. 19.

42 A. Molak, Szkoła pracy Kerschensteinera. Model szkoły dla potrzeb cywilizacji technicznej w społeczeństwie kapitalistycznym, Warszawa 1968; J. Kerschensteiner, Pojęcie szkoły pracy; Podstawowy aksjomat procesu kształcenia, wstęp, przekład i opracowanie B. Nawroczyński, Wrocław-Warszawa-Kraków 1970. 
Im Jahre 1964 gab der staatliche Bildungsverlag Geschichte der Erziehungswissenschaft und des Pädagogikgedankens im Überblick von Stefan Wołoszyn heraus. Der Autor wollte, wie er im Vorwort informierte, die Geschichte der Erziehungswissenschaft „im Zusammenhang mit der Entwicklung der Produktionskraft und den Etappen des Klassenkampfes, besonders darauf hinweisend, wie auf dem Gebiet der Bildung und Erziehung sich in der Bestrebung der unterdrückten Klassen und ihr Kampf um Fortschritt, äußern" ${ }^{\text {"3 }}$ darstellen. Der Autor bemerkte in der Pädagogik Herbarts eine Innovation in der Wissenschaftstheorie des Lernens und Erziehens, für wertvoll empfand er seine Theorie des vielseitigen Interesses, der besonderen Analyse von Lernprozessen. Wołoszyns Einwand weckte dagegen der übertriebene Formalismus und Rigorismus der formalen Stufen, willkürlicher Vorbehaltlosigkeit im Lernen des zu Lernenden. Gemäß der Leitlinien der marxistischen Historiographie suchte der polnische Autor in der Pädagogik Herbarts gesellschaftspolitische Inhalte. Als nicht zu sehr berechtigt fand er, dass seine Pädagogiktheorie „bequem für das herrschende Regime und für die preußische Aristokratie war. Sie rechtfertigte die Gesellschaftshierarchie, das gesellschaftliche Zusammenleben stützte sich vor allem auf das „moralische” Zusammenleben der Einheiten mit den Einheiten (sie propagierte das individualistische Haupterziehungsziel), dessen Grundlage die persönliche Güte der Oberen in Bezug auf die Untergebenen war. An der Spitze dieser Hierarchie stand die persönliche Güte des Monarchen". ${ }^{44}$

In der Mitte der 60er Jahre des vergangenen Jahrhunderts wurde das Lehrbuch Erziehungsgeschichte unter der Leitung von Łukasz Kurdybacha erstellt und in die Hände der Leser übergeben. ${ }^{45}$ Im Lehrbuch wird beurteilt, dass die Lerntheorie von Herbart die wissenschaftliche Reflektion über die Erziehung bedeutend vertiefte. Diese Anerkennung weckte das Interesse von Autoren vielseitiger Theorien. Ersichtlich ist ebenso die Armut des durch die Herbartisten empfohlenen Lernprozesses: „ihre Anstrengungen, damit jede Unterrichtsstunde, mindestens jede methodische Einheit, gemäß formaler Schritte, zur Verfestigung des Lernens durchgeführt wird [...] Schema und Formalismus im Lernen verdrängte die Initiative der Lehrer, bremste die Entwicklung der Fähigkeiten der

43 S. Wołoszyn, Dzieje wychowania i myśli pedagogicznej w zarysie, Warszawa 1964, p. 15.

${ }^{44}$ S. Wołoszyn, Dzieje wychowania i myśli pedagogicznej w zarysie, Warszawa 1964, p. 275.

45 Ł. Kurdybacha, Przedmowa, in: Historia wychowania, t. 1, ed. Ł. Kurdybacha, Warszawa 1965, p. 7. 
Schüler, erschwerte das eigenständige Ausdiskutieren des Lehrmaterials, enge Anpassung an die formalen Schritte beim Lernen beantwortetes kontrollierendes Leben der preußischen Schuladministration" ${ }^{46}$ In polnischen Publikationen über Herbart aus Zeiten der Volksrepublik Polen, also den gemäß der Leitlinien der marxistischen Historiographie geschriebenen, wurden Fakten über die Entwicklung der Bildungsideale im neunzehnten Jahrhundert und dem staatlichen Eingriff in die Bildung ungenau angegeben.

Die pädagogischen Ideen von Herbart wurden unter den polnischen Lesern durch den Kenner der herbartischen Pädagogik Bogdan Nawroczyński ${ }^{47}$ populär. Seiner Meinung nach war die Erfolgsgeschichte der herbartischen Pädagogik "außergewöhnlich" und seine Pionieridee wurde nicht immer richtig zu schätzen gewusst. Im „Pädagogischen Quartal“ aus dem Jahre 1996 bemühte er sich, die Leser auf die pädagogischen Effekte des Werks Herbarts aufmerksam zu machen und hob hervor, dass sein Verdienst unter anderem die Feststellung ist, dass die Pädagogik eine eigenständige Wissenschaft ist. ${ }^{48}$ Nawroczyński erarbeitete den pädagogischen Brief Herbarts, ausgegeben von Ossolineum im Jahre 1967. Nawroczyński besprach das pädagogische System Herbarts, seine Überlegungen zum Thema Methoden des Lernens als „besonders bedeutend” anerkennend. Das Vorwort der Schrift beendete Nawroczyński mit den Worten: „Wir haben das System eines hervorragenden Wissenschaftlers, unermüdlichen Denkers, talentierten Pädagogen kennengelernt. In ihm sind wertvolle Ansichten über Erziehung und noch um einiges wertvollere über das Lernen, aber der größte Verdienst Herbarts ist der, dass er so eifrig arbeitete, um die Pädagogik, die er vorfand, auf wissenschaftliches Niveau zu bringen"49. Die Reichhaltigkeit in der besonderen Publikation Nawroczyńskis zeigte das gesamte Spektrum der Rätsel, zusammenhängend mit dem Herbartizismus, präsentieren mit Macht seine Wirkung auf den pädagogischen Gedanken anderer Autoren.

Eine weitere Arbeit über Herbart, die in Polen zur Zeit der politischen Indoktrination erschien, verbreitete Informationen über sein Leben und seine

46 Historia wychowania, t. 2, ed. Ł. Kurdybacha, Warszawa 1967, pp. 300-302.

47 B. Nawroczyński, Drogi i bezdroża J. F. Herbarta. W 190 rocznicę urodzin (1776-1841), „Nowa Szkoła” (1966), no 11, pp. 26-27.

48 B. Nawroczyński, Kariera pedagogiki J. Fr. Herbarta, „Kwartalnik Pedagogiczny” (1966), no 4, p. 17.

49 B. Nawroczyński, Przedmowa, in: J. F. Herbart, Pisma pedagogiczne, tł. B. Nawroczyński i T. Stera, ed. B. Nawroczyński, Wrocław-Warszawa-Kraków 1967, p. LXXII. 
pädagogische Arbeit. ${ }^{50}$ Dennoch vollbrachte kein Autor eine so ausführliche Analyse des Herbartizismus wie Nawroczyński. Am Ende der achtziger Jahre des vergangenen Jahrhunderts machte Ryszard Wroczyński im Buch Die allgemeine Geschichte des Sportunterrichtes auf die Einkreisung durch Herbart und die Schüler seiner Überlegungen zur Frage der erzieherisch didaktischen Schulen aufmerksam. Den polnischen Autor störte die Umgehung der Fragen der Entwicklung und des Sportunterrichtes bei Jugendlichen durch die herbartische Schule: „Es war zweifelsfrei einer der wichtigen Faktoren des gemächlichen Fortschrittes des Sportunterrichtes in der Schule, vor allem in der Mittelschule, bemächtigte sich noch den Einflüssen des Herbartismus zu Beginn des 20. Jahrhunderts". ${ }^{51}$ Wroczyński verwies auf die englische Schule als einen Ort, in dem im 19. Jahrhundert genauso viele Bemühungen zur physischen Aktivität von Jugendlichen unternommen wurden.

\section{Aktuelle Zeiten}

Der Zusammenbruch der Sowjetunion leitete schnelle Veränderungen in den mittel-osteuropäischen Staaten ein. Die Erlangung der vollen staatlichen Unabhängigkeit durch Polen eröffnete eine neue Etappe in der wissenschaftlichen Forschung. Im letzten Vierteljahrhundert entfalteten sich in Polen die Humanund Gesellschaftswissenschaften, die Anzahl der hauptberuflichen Historiker und Pädagogen stieg an. Die Forschungen an der Bildungsgeschichte werden von Mitarbeitern aller Hochschulen und wissenschaftlicher Institute durchgeführt. Ihr Resultat sind zahlreiche wissenschaftliche Arbeiten. Ein Teil von ihnen betrifft Herbart. Die wissenschaftlichen Forschungen in diesem Zeitraum zeugen von dem Bedarf an nötigen und unnötigen Luken in der polnischen historischen und pädagogischen Literatur.

Frühere Lexikonquellen erinnern, dass Herbart sein pädagogisches System auf Grundlage der psychologische Ethik, die er „wissenschaftliche Pädagogik

${ }^{50}$ K. Sośnicki, Istota i cele wychowania, Warszawa 1964, p. 37; Z. Marciniak, Zarys historii wychowania, Warszawa 1978, pp. 105-106; J. Krasuski, Historia wychowania, Warszawa 1985, p. 119.

${ }^{51}$ R. Wroczyński, Powszechne dzieje wychowania fizycznego i sportu, Wrocław-Warszawa-Kraków-Gdańsk-Łódź 1985, p. 249. 
nannte" aufbaute. ${ }^{52}$ Dem Ziel der Popularisierung des Wissens über Herbart dienen Übersetzungen seiner Arbeiten. Man veröffentlicht sie in historisch-pädagogischen Zeitschriften ${ }^{53}$ und in Form literarischer Werke. ${ }^{54}$ Die Ansichten des deutschen Pädagogen werden in den neuesten Lehrbüchern der Erziehungsgeschichte für Studenten pädagogischer Studienrichtungen zur Pflichtlektüre. Kalina Bartnicka und Irena Szybiak erklären im Lehrbuch Umriss der Erziehungstheorie verständlich die Idee der Erziehungslehre von Herbart, daran erinnernd, dass der deutsche Pädagoge in die Kraft der Erziehung, den Bedarf der Formung starker Charaktere der zu Erziehenden investierte. ${ }^{55}$

Herbarts pädagogische Ansichten bespricht Stefan Możdżeń - Autor des dreibändigen Lehrbuchs zur Erziehungsgeschichte. Im zweiten Band stellt er unterschiedliche bildungs-erzieherische Probleme des 19. Jahrhunderts dar, ausführlich schreibt er über Herbart. Am Ende seiner Betrachtungen äußert er die Gewissheit, dass die Überzeugungen der Herbartisten über die große Bedeutung des Lernens für die Erziehung falsch waren. ${ }^{56}$ Eine weniger ambitionierte Auffassung von Herbarts pädagogischen Gedanken vertritt das Lehrbuch von Mirosław Krajewski unter dem Titel Erziehungsgeschichte und Pädagogikgedanke. Eine Vorlesungsübersicht. ${ }^{57}$ Das neueste akademische Lehrbuch ist das Werk von Czesław Kupisiewicz mit dem Titel: Aus theoretischen und praktischen Vorgängen der Erziehung. Der polnische Autor übermittelt, dass solche Denker, wie Johann Heinrich Pestalozzi, Jean-Jacques Rousseau, der Philosoph Johann Gottlieb Fichte sowie die Neohumanisten Friedrich August Wolf und Wilhelm von Humboldt Einfluss auf die Bildungskonzepte Herbarts nahmen. Kupisiewicz liefert den Lesern das Wissen über die Schüler und

${ }^{52}$ M. Grzywna, Herbart Johann Friedrich (1776-1841), in: Encyklopedia pedagogiczna XXI wieku, t. 2, Warszawa 2003, pp. 183-184; W. Okoń, Nowy słownik pedagogiczny, Warszawa 2007, p. 133.

${ }^{53}$ J. F. Herbart, Estetyczne przedstawienie świata jako główne zajęcie wychowania, tł. D. Stępkowski, „Pedagogika Kultury” (2008), no 4, pp. 13-27; J. F. Herbart, Listy pedagogiczne albo listy o zastosowaniu psychologii w pedagogice, tł. B. Stępkowski, „Przegląd Historyczno-Oświatowy” (2008), no 3-4, pp. 140-147; J. F. Herbart, Wychowanie przy publicznym wspótudziale, tł. D. Stępkowski, „Przegląd Historyczno-Oświatowy” (2008), no 3-4, pp. 151-155.

54 J. F. Herbart, Pedagogika ogólna wywiedziona z celu wychowania, tł. T. Stera, przedmowa i opracowanie D. Stępkowski, Warszawa 2007.

${ }^{55}$ K. Bartnicka, I. Szybiak, Zarys historii wychowania, Warszawa 2001, p. 169.

56 S. I. Możdżeń, Historia wychowania 1795-1918, p. 62.

${ }^{57}$ M. Krajewski, Historia wychowania i myśli pedagogicznej. Zarys wykładu, Płock 2006. 
Anhänger des Pädagogikgedankens von Herbart. Charakterisiert wurden die folgenden Herbartisten: Tuiscon Ziller, Karl Stoy, Otto Willmann und Wilhelm Rein. Der Autor des Lehrbuches meint, dass trotz der Unvollkommenheit des Konzeptes Herbarts formaler Schritte und seiner Epigone, „ein bedeutender Schritt in Bezug auf die früheren Versuche der Erziehung, besonders des Lernens", ${ }^{58}$ waren.

Die Popularisierung des Wissens über Herbart und das Erregen des Interesses an deutscher Pädagogik dienen der Wissenschaftskonferenz. Im Herbst des Jahres 2006 fand in Warschau eine Konferenz unter dem Titel Herbart bekannt und unbekannt. Zum zweihundertsten Geburtstag der Ausgabe der allgemeinen Pädagogik ${ }^{59}$ statt. Ihre Nachlese ist ein Buch, welches das während der Konferenz gehaltene Referat beinhaltet. Im Vorwort steht geschrieben: „Herbarts Beitrag und später der seiner Anhänger - bezeichnet mit dem Namen Herbartisten - in der Entwicklung der neuzeitlichen Pädagogik ist Gegenstand vieler Kontroversen und widersprüchlicher Bewertungen. Für unbestreitbar ist dennoch anzuerkennen, dass Die allgemeine Pädagogik ein neues Kapitel nicht nur in Werken der Erziehung, aber auch in wissenschaftlichen Reflexionen über sie ist. Bis jetzt wurde dies in der polnischen Pädagogik weder ausreichend bemerkt, noch eindeutig hervorgehoben. Daher ist der Jubiläum ein passender Anlass, um sowohl an den deutschen Philosoph, als auch an sein erstes Werk zu erinnern". ${ }^{60}$ Während der Konferenz wurde unter anderem über Herbart als Weiterführer des alten Pädagogikgedankens, die Aufnahme der herbartischen Konzeption der „Wissenschaftlichkeit“ der Pädagogik, herbartische Inspirationen für die gegenwärtige Pädagogik diskutiert.

Einen bedeutenden Beitrag in der Verbreitung des Wissens über Herbart erbrachte Andrzej Murzyn. Im Jahre 2004 erschien sein Buch Johann Friedrich Herbart und sein Platz im Kontext der Exekution des Idealismusgedankens. ${ }^{61}$ Einige Jahre später gab er die wertvolle Arbeit unter dem Titel J. F. Herbarts Bildungsund Erziehungsphilosophie heraus. Sie ist die Pionierprobe der Aufnahme der

${ }^{58}$ C. Kupisiewicz, Z dziejów teorii i praktyki wychowania. Podręcznik akademicki, Kraków 2012, p. 191.

${ }^{59}$ D. Stępkowski, Inspiracje herbartowskie współczesnej pedagogiki (głosy uczestników dyskusji panelowej), „Seminare” (2008), t. 25, pp. 389-402.

${ }^{60}$ Herbart znany i nieznany. W dwusetna rocznicę wydania Pedagogiki ogólnej (1806-2006), eds. J. Piskurewicz i D. Stępkowski, Warszawa 2006, p. 9.

${ }^{61}$ A. Murzyn, Johann Friedrich Herbart i jego miejsce w kontekście pokantowskiej myśli idealistycznej, Kraków 2004. 
Gesamtheit der Problematik bezüglich der Lern- und Erziehungskonzeption durch den deutschen Pädagogen. Der Autor verweist auf die Quelle der philosophisch-pädagogischen Inspirationen Herbarts, er bespricht die Regeln der wissenschaftlichen Pädagogik. Am Ende meinte er: „Falls wir - wenigstes zum Teil - vorhaben, den Sinn der Erziehungslehre Herbarts herauszulesen, müssen wir daran denken, dass das Werk dieses großen Denkers auf der Suche nach pädagogischer Philosophie und philosophischer Pädagogik geboren wurde [...] Herbart war ganz bewusst und konsequent zu beweisen, dass die Lehre immer eine dienliche Funktion gegenüber der Erziehung erfüllt, welche die Entwicklung der Moralvorstellung und zugleich des Ethischen ist". ${ }^{62}$ Im besprochenen Buch wurde eine Bibliografie mit einem Verzeichnis verschiedensprachiger Publikationen über die herbartische Pädagogik veröffentlicht.

Das aktuelle Interesse am Herbartismus in Polen ist Zeugnis der bleibenden Bedeutung Herbarts pädagogischer Theorie. Trotz dessen, dass ihre Grundsätze in der aktuellen pädagogischen Praxis schon keine Priorität mehr sind, schreibt man ihnen eine inspirierende Rolle in der Betrachtung der Erziehungsgeschichte $\mathrm{zu}$, sowie in der Reflexion der Entwicklung der pionierartigen Bildungsund Erziehungsidee, welche in einem gewissen Zeitraum das Bemessen des Schulwesens veränderten. Jetzige Forschungen an Herbarts Pädagogikgedanken trugen ihre Früchte in Abhandlungen bedeutenden Inhalts. Unter diesen Arbeiten sind Werke, für welche deren Autoren einen Doktortitel oder den Titel eines habilitierten Doktors erhielten. Dies bezeugt gewissermaßen die Meinung der Wissenschaftler, dass der Wissenschaftsbereich der Erkenntnis des pädagogischen Gedankens unbegrenzt ist.

Am Ende der Betrachtungen über die Rezeption der Pädagogikgedanken Herbarts in Polen sollte daran erinnert werden, dass das Interesse an der Pädagogik Herbarts in Polen sich in besonderen historischen Zeiträumen unterschiedlich intensiv gestaltete und von vielen Faktoren abhängig war. Ende des 19. Jahrhunderts bemühte man sich, die bildungserzieherische Idee Herbarts praktisch in polnischen Schulen im österreichisch besetzten Gebiet anzuwenden. Ein Teil der Lehrer glaubte, dass das erzieherische Lehren im Charakter des Schülers positive Veränderungen hervorruft und dass Didaktik auf Grundlage formaler Schritte einen Lernerfolg garantiert. Die polnische Literatur aus dieser Zeit enthält in vielen Fällen ein kritikloses Lob des Herbartismus. Die Ausgabe des Werkes Pädagogisches Allgemeinwissen mit dem Ziel der Erziehung

62 A. Murzyn, Filozofia nauczania wychowującego J. F. Herbarta, Kraków 2010, p. 111. 
in polnischer Sprache ermöglichte dem polnischen Pädagogen, der die deutsche Sprache nicht beherrschten, Zugang zur Lektüre mit den Betrachtungen Herbarts zu haben. Erziehungshistoriker haben keinen Zweifel, dass die Popularisierung des Herbartismus mit Hilfe der Übersetzungen deutscher Pädagogikwerke „die Entwicklung der Pädagogik als universitäre Disziplin bewirkte". ${ }^{63}$

Im Zeitraum zwischen dem Ersten und dem Zweiten Weltkrieg bereicherte sich die polnische pädagogikhistorische Literatur um die Publikationen weiterer herbartischer Artikel. Sie sind berühmte Zeugnisse des Glaubens polnischer Pädagogen an die Möglichkeit den Herbartismus in der erzieherischen Praxis anzuwenden. Immer öfter schrieb man damals über die Unvollkommenheit der Pädagogiktheorie Herbarts. Der damalige Pädagogikgedanke wurde mit den Bildungsgrundsätzen neuer Pädagogikrichtungen konfrontiert, die sich in den ersten Jahrzehnten des 20. Jahrhunderts dynamisch entwickelten und ein neues Licht auf Bildungs- und Erziehungsaspekte warfen, welche von Vertretern der sogenannten traditionellen Erziehungsmethode - die sich durch Rigorismus und Diktatur gegenüber Kindern und Jugendlichen auszeichnete - nicht betrachtet wurden.

In den Jahren 1945-1989, also als Polen der sowjetischen Einflusszone angehörte, wurde die herbartische Pädagogik nicht so intensiv publiziert, wie die pädagogischen Konzeptionen sowjetischer Autoren. In diesem nicht einfachen Zeitraum entstanden großartige polnische Veröffentlichungen, die das Wesen der Bildungsansichten Herbarts darstellen. Eine merkliche Rolle in Sachen ihrer Publikation spielte B. Nawroczyński. Man darf dennoch nicht verschweigen, dass ein Teil der damals in Polen herausgegebenen Arbeiten über Herbart falsch interpretierte Grundsätze seiner Pädagogik enthielt - sie zählten ihn zum Kreis der Bourgeoisepädagogen.

In den letzten Jahren zeigten sich am polnischen Buchmarkt viele literarische Publikationen und Wissenschaftsartikel, die die Erziehungsgeschichte betreffen. Ein Teil von ihnen bezieht sich auf Herbarts pädagogischen Gedanken. Man diskutiert über ihn auch auf wissenschaftlichen Konferenzen. An der Diskussion nehmen nicht nur Polen teil, sondern auch ausländische Spezialisten. Das Interesse am Herbartismus ist von unbestrittener Wichtigkeit. Davon zeugt die Zahl von Autoren und Publikationen. Es ist zu vermuten, dass das Interesse am deutschen Pädagogikgedanken in den nächsten Jahren nicht abnimmt, sondern dass er sich intensiv entwickelt und man ihn besser kennen lernen können wird.

${ }^{63}$ S. I. Możdżeń, Historia wychowania 1795-1918, p. 333. 


\section{Bibliography}

Baranowski M., Dydaktyka do użytku seminariów nauczycielskich i nauczycieli szkót wyższych, Lwów 1895.

Bartnicka K., Szybiak I., Zarys historii wychowania, Warszawa 2001.

Chmaj L., Kierunki i prądy pedagogiki współczesnej, Warszawa-Wilno 1938.

Danysz A., O kształceniu, Lwów 1918.

Danysz A., O wychowaniu, Lwów 1903.

Dybiec J., Polska w orbicie wielkich idei: polskie przekłady obcojęzycznego piśmiennictwa 1795-1918, t. 1, Warszawa 2011.

Dybiec J., Recepcja idei pedagogicznych w Polsce, in: Recepcja w Polsce nowych kierunków i teorii naukowych, ed. A. Strzałkowski, Kraków 2001, pp. 195-202.

Gara J., Specyfika polskiej recepcji twórczości Johanna Friedricha Herbarta, „Przegląd Pedagogiczny" (2012), no 2, pp. 22-31.

Grzywna M., Herbart Johann Friedrich (1776-1841), in: Encyklopedia pedagogiczna XXI wieku, t. 2, Warszawa 2003, pp. 183-184.

Herbart J. F., Wychowanie przy publicznym współudziale, tł. D. Stępkowski, „Przegląd Historyczno-Oświatowy" (2008), no 3-4, pp. 151-155.

Herbart J. F., Estetyczne przedstawienie świata jako główne zajęcie wychowania, tł. D. Stępkowski, „Pedagogika Kultury” (2008), no 4, pp. 13-27.

Herbart J. F., Listy pedagogiczne albo listy o zastosowaniu psychologii w pedagogice, tł. B. Stępkowski, „Przegląd Historyczno-Oświatowy” (2008), no 3-4, pp. 140-147.

Herbart J. F., Pedagogika ogólna wywiedziona z celu wychowania, t1. T. Stera, Warszawa 1912.

Herbart J. F., Pedagogika ogólna wywiedziona z celu wychowania, tł. T. Stera, przedmowa i opracowanie D. Stępkowski, Warszawa 2007.

Herbart J. F., Pisma pedagogiczne, tł. B. Nawroczyński i T. Stera, ed. B. Nawroczyński, Wrocław-Warszawa-Kraków 1967.

Herbart J. F., Wykłady pedagogiczne w zarysie, tł. J. Jakóbiec, przejrzał F. Bielak, Warszawa 1937.

Herbart znany i nieznany. W dwusetną rocznicę wydania Pedagogiki ogólnej (1806-2006), eds. J. Piskurewicz i D. Stępkowski, Warszawa 2006.

Historia wychowania, t. 2, ed. Ł. Kurdybacha, Warszawa 1967.

Kerschensteiner J., Pojęcie szkoły pracy; Podstawowy aksjomat procesu kształcenia, wstęp, przekład i opracowanie B. Nawroczyński, Wrocław-Warszawa-Kraków 1970.

Kierski F., Podręczna encyklopedia pedagogiczna, t. 1, Lwów-Warszawa 1923.

Kostkiewicz J., Kierunki i koncepcje pedagogiki katolickiej w Polsce 1918-1939, Kraków 2013.

Kot S., Historia wychowania, t. 2, Lwów 1934. 
Koźmian D., Chrześcijańska myśl wychowawcza Fryderyka Wilhelma Foerstera i jej recepcja $w$ Polsce, Szczecin 1996.

Krajewski M., Historia wychowania i myśli pedagogicznej. Zarys wykładu, Płock 2006.

Krasuski J., Historia wychowania, Warszawa 1985.

Krupa M., Fryderyk Adolf Diesterweg i jego wplyw na polska pedagogikę w XIX wieku, Wrocław 1976.

Kulczyński L., Herbart Jan Fryderyk, in: Encyklopedia wychowawcza, t. 5, Warszawa 1901, pp. 267-275.

Kupisiewicz C., Z dziejów teorii i praktyki wychowania. Podręcznik akademicki, Kraków 2012.

Łobocki M., Teoria wychowania w zarysie, Kraków 2010.

Majchrowicz F., Historia pedagogii dla użytku seminariów nauczycielskich i nauki prywatnej, t. 2, Lwów 1920.

Majorek C., System kształcenia nauczycieli szkółludowych w Galicji doby autonomicznej 1871-1914, Wrocław 1971.

Marciniak Z., Zarys historii wychowania, Warszawa 1978.

Molak A., Szkoła pracy Kerschensteinera. Model szkoły dla potrzeb cywilizacji technicznej w społeczeństwie kapitalistycznym, Warszawa 1968.

Możdżeń S. I., Historia wychowania 1795-1918, Sandomierz 2006.

Możdżeń S. I., Historia wychowania 1918-1945, Sandomierz 2006.

Murzyn A., Johann Friedrich Herbart i jego miejsce w kontekście pokantowskiej myśli idealistycznej, Kraków 2004.

Murzyn A., Filozofia nauczania wychowującego J. F. Herbarta, Kraków 2010.

Nawroczyński B., Drogi i bezdroża J. F. Herbarta. W 190 rocznicę urodzin (1776-1841), „Nowa Szkoła” (1966), no 11, pp. 26-27.

Nawroczyński B., Kariera pedagogiki J. Fr. Herbarta, „Kwartalnik Pedagogiczny” (1966), no 4, pp. 17-25.

Nawroczyński B., Polska myśl pedagogiczna, Lwów -Warszawa 1938.

Nawroczyński B., Zasady nauczania, Lwów-Warszawa 1930.

Okoń W., Nowy słownik pedagogiczny, Warszawa 2007.

Seredyński W., Tekst do wykładu pedagogiki. Wykład metody nauk realnych $w$ szkole ludowej dla użytku seminariów nauczycielskich, Tarnów 1888.

Sękowski M., Spencer i pozytywizm warszawski, Lublin 1986.

Sobczak J., Recepcja idei nowego wychowania w polskiej pedagogice okresu między wojnami, Bydgoszcz 1979.

Sośnicki K., Dydaktyka ogólna, in: Encyklopedia wychowania, t. 2, Warszawa 1937.

Sośnicki K., Istota i cele wychowania, Warszawa 1964.

Sośnicki K., Rozwój pedagogiki zachodniej na przełomie XIX i XX wieku, Warszawa 1967. 
Stępkowski D., Herbart i jego myśl w Polsce. Dzieje recepcji i oddziaływań, „Artes liberales. Zeszyty Naukowe Akademii Humanistycznej im. A. Gieysztora” (2007), no 1, pp. 1-33.

Stępkowski D., Inspiracje herbartowskie współczesnej pedagogiki (głosy uczestników dyskusji panelowej), „Seminare” (2008), t. 25, pp. 389-402.

Szulakiewicz W., Historia oświaty $i$ wychowania w Polsce 1918-1939. Studium historiograficzne, Toruń 2000.

Szulakiewicz W., Historia oświaty i wychowania w Polsce 1944-1956, Kraków 2006.

Trentowski B. F., Chowanna, czyli system pedagogiki narodowej jako umiejętności wychowania, nauki i oświaty, słowem wykształcenia naszej młodzieży, t. 1-2, ed. A. Walicki, Wrocław-Warszawa-Kraków 1970.

Troskolański T., Zasługi Herbarta około wychowania, Lwów 1899.

Wernic H., Myśli o wychowaniu i nauczaniu. Z autobiografia i portretem autora, Warszawa 1895.

Wnęk J., Polskie przekłady literatury zagranicznej 1918-1939, Kraków 2006.

Wołoszyn S., Nauki o wychowaniu w Polsce w XX wieku. Próba syntetycznego zarysu na tle powszechnym, Kielce 1998.

Wołoszyn, S. Dzieje wychowania i myśli pedagogicznej w zarysie, Warszawa 1964.

Woroniecki J., Katolicka etyka wychowawcza, Poznań-Warszawa-Wilno-Lublin 1925.

Wroczyński R., Powszechne dzieje wychowania fizycznego i sportu, Wrocław-Warszawa-Kraków-Gdańsk-Łódź 1985.

Z dziejów myśli pedagogicznej. Materiały do studiów, eds. B. Suchodolski, W. Okoń, Warszawa 1958.

Zarzecki L., Charakter jako cel wychowania, Warszawa 1918. 